\title{
Longterm X-ray observations of blazars with MAXI
}

\author{
Isobe Naoki ${ }^{1}$ and the MAXI team. \\ ${ }^{1}$ Department of Astronomy, Kyoto University \\ email: n-isobe@kusastro.kyoto-u.ac.jp
}

\begin{abstract}
Longterm continuous X-ray observations of blazars with MAXI are reported. Thanks to its unprecedentedly high sensitivity as an all sky X-ray monitor, MAXI is an ideal observatory to investigate variability of blazars, which should give a clue to particle acceleration in their jet, as well as the jet dynamics. Actually, since it started its operation in the summer of 2009, MAXI has successfully alerted two strong X-ray flares from the BL Lac object Mrk 421. Especially, in one of these flares, the X-ray flux of the object was found to become the highest in history. By closely examining the MAXI data, the physical quantities associated with the flares were estimated. These results clearly demonstrate the potential of MAXI for the variability of blazars.
\end{abstract}

Keywords. galaxies: jets, BL Lacertae objects: individual (Mrk 421), X-rays: galaxies

\section{Introduction}

One of the most outstanding properties of blazars is their rapid and high-amplitude intensity variation or flares, which is thought to be a useful probe for the flow dynamics, as well as to particle acceleration and cooling processes operating in their jet. Since the Ginga and ASCA era, X-ray observations have been a valuable tool for the blazar variability. Recently, based on the monitoring X-ray observations with the RXTE ASM and Swift, X-ray flares were successively detected from a number of blazars.

Since it was mounted on the Japanese Experiment Module "Kibo" of the International Space Station (ISS) in the summer of 2009, Monitor of All-sky X-ray Image (MAXI; Matsuoka et al. 2009) has been conducting successful all sky X-ray observations for more than one year. MAXI has the highest sensitivity as an all sky X-ray monitor, together with a high sky coverage ( $\sim 95 \%$ per day). These make this instrument the ideal observatory to investigate the longterm variability of blazars. Actually, MAXI has successfully made quick alerts of two strong X-ray flares from the High energy Peaked BL Lac object (HBL) Mrk 421 (Isobe et al. 2010a, 2010b). Currently, the MAXI lightcurve of more than 10 blazars are available from the MAXI web site (http://maxi.riken.jp/top/index.php).

\section{Results}

As shown in the $2-10 \mathrm{keV}$ MAXI lightcurve of Mrk 421 in Figure 1 (1), the object was found to be highly variable. Especially, the MAXI has detected at least four X-ray flares with an X-ray flux exceeding the $100 \mathrm{mCrab}$ level $\left(2 \times 10^{-9} \mathrm{ergs} \mathrm{cm}^{-2} \mathrm{~s}^{-1}\right.$ in $\left.2-10 \mathrm{keV}\right)$. Due to relatively bad observational conditions for the first and second flares, we concentrate on the data associated with the third and forth ones on 2010 January 1 (MJD = 55136; Isobe et al. 2010a) and 2010 February 17 (MJD = 55146; Isobe et al. 2010b), respectively. These data are shown in Figure 1 (2) and (3), denoted as Epochs A and B. The maximum 2-10 keV flux in Epochs A and B was measured as $120 \pm 10 \mathrm{mCrab}$ at $\mathrm{MJD}=55197.4$ and $164 \pm 17 \mathrm{mCrab}$ at $\mathrm{MJD}=55243.6$, respectively. Especially, 


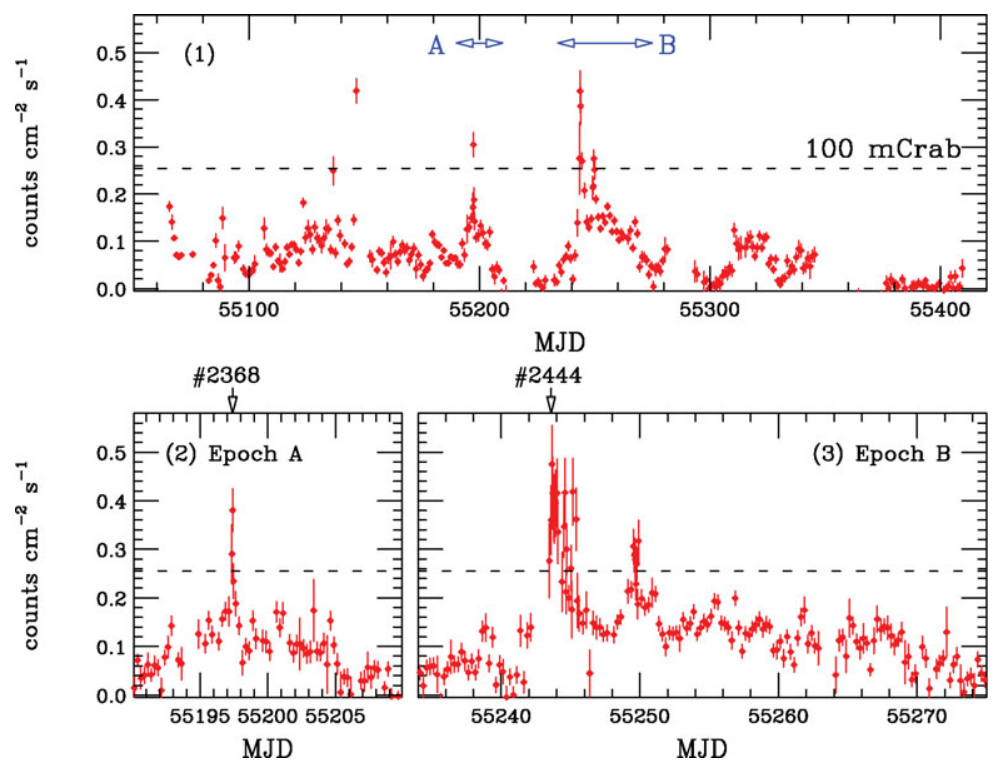

Figure 1. (1) Daily averaged $2-10 \mathrm{keV}$ MAXI lightcurve of Mrk 421 from 2009 August 22 $(\mathrm{MJD}=55062)$ to 2010 August $1(\mathrm{MJD}=55409)$. The count rate for $100 \mathrm{mCrab}$ is indicated by the dashed line. The data corresponding to the two arrows, denoted as A and B, are expanded to the panels (2) and (3), respectively. The two strong X-ray flares, alerted by MAXI (Isobe et al. 2010a, 2010b), are shown by the arrows on the top of the panels (2) and (3) with the article number on the Astronomer's Telegram.

the latter is found to be the highest among those ever reported from the objects (e.g., $\sim 130$ mCrab in 2008 June; Donnarumma et al. 2009). Therefore, we have concluded that MAXI discovered the strongest X-ray flare from Mrk 421. In addition, this event is probably concurrent with a strong very high energy $\gamma$-ray $(>100 \mathrm{GeV})$ flare with a flux of $>10 \mathrm{Crab}$, detected by the VERITAS Observatory (Ong et al. 2010).

Assuming that the decay time $t_{\mathrm{d}}$ of the flare is determined by the cooling timescale due to the synchrotron radiation, the dominant cooling process in typical HBLs, the magnetic field strength $B$ is roughly estimated. For the flare in Epoch A, the decay time, $t_{\mathrm{d}}=2.5 \times 10^{4} \mathrm{~s}$, gives a magnetic field of $B=0.045(\delta / 10)^{-1 / 3} \mathrm{G}$ with $\delta$ being the jet beaming factor (Isobe et al. 2010c). This is found to be consistent with the value determined derived in the previous studies (e.g., $B=0.036-0.44 \mathrm{G}$; Kino et al. 2002). In contrast, the decay time longer by a factor of $5-6$ in the Epoch B flare, $t_{\mathrm{d}}=1.3 \times 10^{3}$ $\mathrm{s}$, corresponds to a weaker magnetic field of $B=0.015(\delta / 10)^{-1 / 3} \mathrm{G}$ (Isobe et al. 2010c). We speculate that the Epoch B flare could be not a single event. These results, derived by the high quality continuous lightcurve with MAXI, clearly demonstrate the advantage of MAXI for the variability study of blazars.

\section{References}

Donnarumma, I. et al., 2009, ApJ, 691, 13

Isobe N., et al., 2010a, Astronomer's Telegram \#2368

Isobe N., et al., 2010b, Astronomer's Telegram \#2444

Isobe N., et al., 2010c, PASJ, in press (arXiv:1010.1003)

Kino, M., Takahara, F., \& Kusunose, M., 2002, ApJ, 564, 97

Matsuoka, M., et al. 2009, PASJ, 61, 999

Ong, R. A., et al., 2010, Astronomer's Telegram, 2443 